\title{
Realization of a Biped Robot Lower Limb Walking without Double Support Phase on Uneven Terrain
}

\author{
Hai-yan Wang and Yi-bin Li \\ School of Control Science and Engineering, Shandong University, Jinan 250061, China \\ Correspondence should be addressed to Yi-bin Li; liyb1960@yeah.net
}

Received 19 May 2013; Revised 24 July 2013; Accepted 31 July 2013

Academic Editor: James Lam

Copyright (C) 2013 H.-y. Wang and Y.-b. Li. This is an open access article distributed under the Creative Commons Attribution License, which permits unrestricted use, distribution, and reproduction in any medium, provided the original work is properly cited.

Zero moment point (ZMP) is widely used in dynamical walking control of the biped robot, but it is hard to obtain the ZMP exactly. The paper describes a simple walking control method without using ZMP information directly. Firstly, the paper introduced a biped robot lower-limb prototype which is driven by linear hydraulic servocylinder. Then the paper simplifies the walking control in the lateral plane with a simple walking pattern generation method named "dynamic equilibrium method," which is fit for active and underactuated biped robots. In the following section the paper provides the balance control methods without using ZMP information directly. Finally, simulation experiments with MD.DAMS and experiments in physical prototype are given. The experimental results confirm the effectiveness of the proposed control methods.

\section{Introduction}

To investigate the stability of human walking, Vukobratovic proposed a concept called zero moment point (ZMP) [1]. Although the ZMP algorithm and its variants have been used to realize stable walking for many bipedal robots, they are somewhat complex and computationally intensive since the full dynamics of the robot is considered. Kajita et al. introduced the single mass linear inverted pendulum model (LIPM), instead of using the complicated model where multibody mass is considered [2]. With three assumed condition, the ZMP equation for an $n$-DOF (degree of freedom) biped robot was simplified. Kajita also brought a successful result in the real experiment, and the maximum walking speed of HRP-2 was $2.5 \mathrm{~km} / \mathrm{h}$ [3]. Kim introduced convolution sum [4], in which the proposed online algorithm has a similarity with the preview control [2] that needs a finite future reference, but it is not required to solve the Riccati equation to get gains. Kim et al. successfully applied this algorithm to the passenger-carrying biped robot HUBO FX1 [5]. The control strategies provided by Kajita et al. and Kim et al. have no difference in the lateral plane and the sagittal plane. Walking pattern generation with LIPM only fits for the biped robot whose mass of legs can be neglected. But there are some robots that do not meet that assumed condition. The paper simplifies the walking control in the lateral plane with a simple walking pattern generation method named "dynamic equilibrium method." It is fit for not only active biped but also underactuated robots, and there is no limit to the mass distribution between the body and the legs.

Biped robots with weight loading capability are different from the general life-size humanoid robots, such as HRP $[4,5]$, ASIMO [6, 7], KHR [8], and BHR [9, 10]. Posture control of the upper body was helpful to realize the stable motion of the life-size humanoid robots. But the biped robots with the weight loading capability only have lower limb and a trunk which could be equipped with scaffolds for the loading or seats for the passenger as the users' needs. The WL-16 has 6-DOF parallel mechanisms and was the first biped walking robot capable of carrying a human in the world $[11,12]$. The weight of this original robot is $56 \mathrm{kgf}$, including $8 \mathrm{kgf}$ battery weight, and the height is approximately $1.2 \mathrm{~m}$. With the same load ability, the robots with electric motor driving system powered by batteries are usually large in volume and heavy in weight. Toyota released their "i-foot" robot in 2004 [13]. The height of the "i-foot" is $2.36 \mathrm{~m}$, with a weight of $200 \mathrm{kgf}$. The maximum weight capacity of passenger was $60 \mathrm{kgf}$. Kim et al. proposed HUBO FX-1 [5] whose height is $1.987 \mathrm{~m}$, with 
TABLE 1: Specifications of the hydraulic biped robot.

\begin{tabular}{lc}
\hline Weight $(\mathrm{kg})$ & Trunk: 12.53 \\
& A single leg: 13.61 \\
\hline \multirow{2}{*}{ Size $(\mathrm{mm})$} & Height in $Z_{c}: 882.36$ \\
& Width in $Y_{c}: 482$ \\
& Length in $X_{c}: 440$ \\
DOF & Hip: 2 \\
& Knee: 1 \\
& Ankle: 1 \\
\hline
\end{tabular}

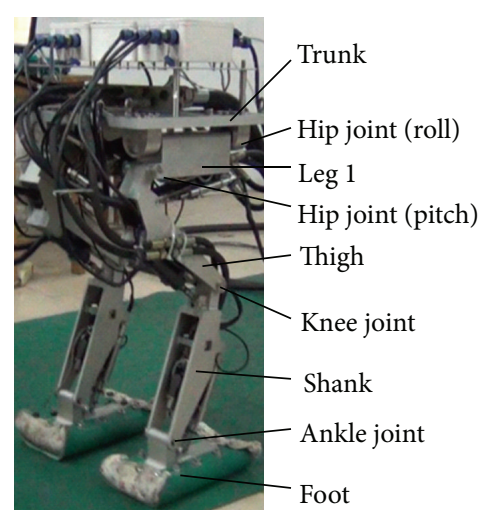

Figure 1: The physical prototype.

a weight of $130 \mathrm{kgf}$ with the exception of the external covers and the cockpit. The maximum weight capacity of passenger is $100 \mathrm{kgf}$.

Compared with electric motor driving system, hydraulic driving system has several important advantages [14], such as higher power-to-mass ratio, larger load capability, greater endurance, faster dynamic response, and better load stiffness. The paper introduced a hydraulic actuated biped robot lower limb with the theoretical maximum load capacity of $200 \mathrm{kgf}$, which is limited to the output force of the hydraulic cylinder. (In ADAMS simulation environment, there is no limit to the weight capability, and then we set a box on the robot trunk which is $100 \mathrm{kgf}$ in weight.) The maximum height of the basic machine is about $882.4 \mathrm{~mm}$, and the weight of the physical prototype robot is about $40 \mathrm{~kg}$, which is mainly used in the no-load test.

\section{Overview of the Hydraulic Actuated Biped Robot Lower Limb}

The hydraulic actuated biped robot lower limb consists of the original robot system and hydraulic driven system. Most humanoid robots has six freedoms in one leg, three in the hip, one in the knee, and two in the ankle [15]. According to the kinetic characteristics of the hydraulic actuated biped robot, the leg structure is simplified here. The biped robot lower limb only has four active freedoms in one leg (shown as Figure 1), two in the hip, one in knee, and one in ankle. The trunk is used to load weight or install scaffold, and there is no freedom on the trunk. Table 1 is the specifications of the hydraulic biped robot. For it is not easy to set the buffer mechanism in the

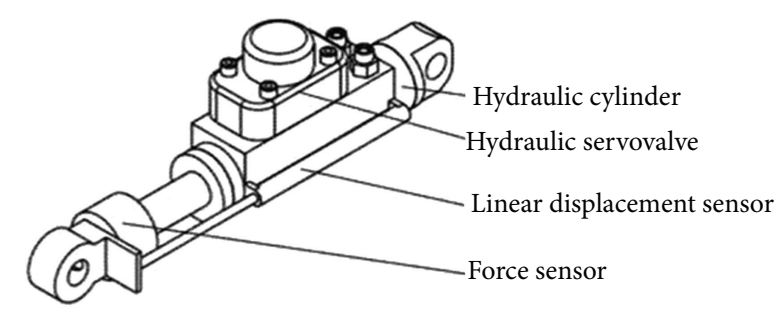

FIGURE 2: Linear hydraulic servocylinder.

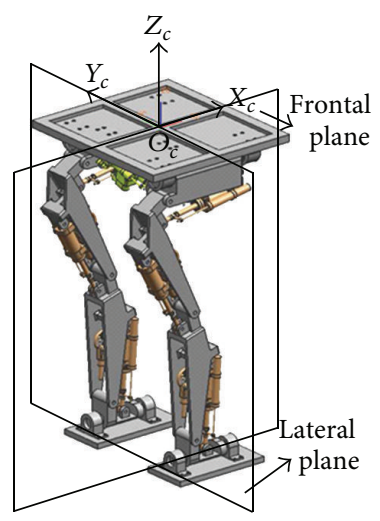

FIGURE 3: The diagram of the motion direction.

ADAMS simulation model, so the rolling joints in the ankles are not locked. Each of them is installed a torsion spring with the stiffness coefficient of $10^{6} \mathrm{~N} \cdot \mathrm{mm} / \mathrm{rad}$ and a rotary damper with the damping coefficient of $10^{6} \mathrm{~N} \cdot \mathrm{mm} \cdot \mathrm{s} / \mathrm{rad}$. The roll angle in the ankle is used to replace the deformation of the buffer mechanism fixed on the foot bottom.

Considering economy problems, all the joints in the biped lower limb are actuated by identical linear hydraulic servocylinder (shown as Figure 2), which is composed of a hydraulic cylinder, a hydraulic servovalve, a linear displacement sensor and a force sensor. The maximum displacement of the linear hydraulic servocylinder is $60 \mathrm{~mm}$.

\section{Walking Pattern Generation}

In the biped robotics research field, the gait trajectory, otherwise known as the walking pattern, generates the relative position trajectories of two feet with respect to the pelvis center [16]. The paper set the geometric center of the trunk upper surface as the origin point of the basic coordinate system; $X_{c}$ points to the direction of forward motion, $Z_{c}$ is perpendicular to the trunk (shown as Figure 3), and then $X_{c} O_{c} Z_{c}$ is the frontal plane and $Y_{c} O_{c} Z_{c}$ is the lateral plane.

3.1. "Mobile Equilibrium Method" in the Lateral Plane. The three-dimensional linear inverted pendulum mode (3DLIPM) combined with zero moment point criterion are widely used in the biped robot control field. But the 3D-LIPM only fits for the biped robots with active ankle joints and whose legs are light compared with the upper part of the body. This paper proposes an easier walking pattern method which 

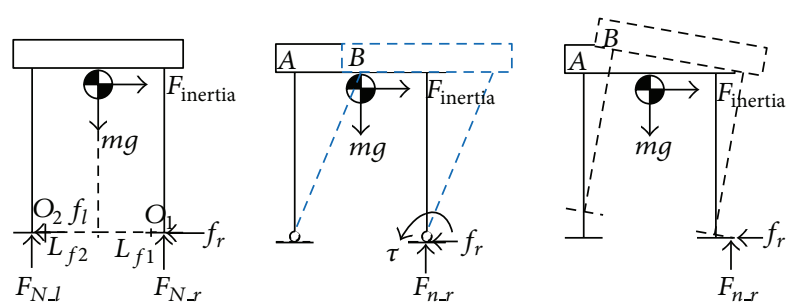

Force analysis with rolling joint in the ankle without rolling joint

FIGURE 4: Force analysis in the lateral plane.

has no limits to the robot mass distribution. It also fits for the robot without active ankle joint in the lateral plane.

We consider the robot's center of mass (COM) as the research object. When feet move accelerated toward left relative to the trunk in the lateral plane, the inertia force in the robot's COM points to right (shown as Figure 4 left). If the acceleration is large enough and the ground can provide enough friction and support force, the force between the left foot and the ground becomes zero, and the trunk moves right. If the roll joint of the ankle in the lateral plane is active, in the ideal condition the trunk moves right without trunk roll motion (shown in Figure 4, middle); if the ankle does not have roll joints, the trunk's roll angle is large when the trunk move right (shown as Figure 4 right); if the ankle has roll joints but they are passive, the trunk's roll angle exists, but it is smaller.

The biped robot lower-limb introduced in this paper does not have roll joint in the ankle, that is to say the roll angle of the trunk must exist when the feet move accelerated to left/right relative to the trunk (suppose that the ground can provide enough friction force, this is the precondition). If the trunk's roll angle changes periodically, it shows that the robot is stable in the lateral plane; otherwise, we should make some adjustments.

When the feet move accelerated to left relative to the trunk with appropriate acceleration, the support force on the left foot decreases to zero, then the left foot lifts, and the trunk will not dump in the lateral plane. The theory is fit for the opposite direction. In order to keep the robot stable in the lateral plane, the following two conditions are needed.

(1) The initial acceleration in $Y_{c}$ direction is adequate. If the acceleration is too small the swing foot cannot lift or the robot topples toward the swing leg; if the acceleration is too large, the robot topples toward the support leg.

(2) In the moving process, the resultant moment is zero which is created by the inertial force and the gravity relative to equilibrium point $O_{1}$ (the equilibrium is $O_{1}$, when the robot COM moves to right; the equilibrium is $\mathrm{O}_{2}$, when the robot COM moves to left. Actually $\mathrm{O}_{1}$ and $\mathrm{O}_{2}$ are zero moment points).

In the above process, the acceleration of the trunk keeps on changing with the displacement changes in the lateral plane, so the method is named as "mobile equilibrium method."
3.2. Walking Pattern Generation in $Y_{c}$ Direction. Two step periods are considered as one movement circle. Based on the "mobile equilibrium method", we can obtain the walking pattern in $Y_{c}$ direction.

In the first step period, the feet move accelerated toward left relative to the trunk.

$$
m g\left(-L_{f 1}-y\right)+m \ddot{y} z_{c}=0 .
$$

Then,

$$
y=c_{1} e^{t \cdot \sqrt{g / z_{c}}}+c_{2} e^{-t \cdot \sqrt{g / z_{c}}}+L_{f 1}
$$

where

$$
\begin{aligned}
& c_{1}=\frac{-e^{-T_{n} \sqrt{g / z_{c}}} \cdot L_{f 1}}{e^{T_{n} \sqrt{g / z_{c}}}-e^{-T_{n} \sqrt{g / z_{c}}}}, \\
& t \in\left[0, T_{n}\right], \\
& c_{2}=\frac{e^{T_{n} \sqrt{g / z_{c}}} \cdot L_{f 1}}{e^{T_{n} \sqrt{g / z_{c}}}-e^{-T_{n} \sqrt{g / z_{c}}}}, \\
& c_{1}=\frac{e^{-T_{n} \sqrt{g / z_{c}}} \cdot L_{f 1}}{e^{T_{c} \sqrt{g / z_{c}}} \cdot e^{-T_{n} \sqrt{g / z_{c}}}-e^{-T_{c} \sqrt{g / z_{c}}} \cdot e^{T_{n} \sqrt{g / z_{c}}}}, \\
& t \in\left[T_{n}, T_{c}\right], \\
& c_{2}=\frac{-e^{T_{n} \sqrt{g / z_{c}}} \cdot L_{f 1}}{e^{T_{c} \sqrt{g / z_{c}}} \cdot e^{-T_{n} \sqrt{g / z_{c}}}-e^{-T_{c} \sqrt{g / z_{c}}} \cdot e^{T_{n} \sqrt{g / z_{c}}} .}
\end{aligned}
$$

In the second step period, the feet move accelerated toward right relative to the trunk:

$$
m g\left(L_{f 2}-y\right)+m \ddot{y} z_{c}=0 .
$$

Then,

$$
y=c_{3} e^{t \cdot \sqrt{g / z_{c}}}+c_{4} e^{-t \cdot \sqrt{g / z_{c}}}-L_{f 2} .
$$

While $t \in\left[T_{c}, T_{c}+T_{n}\right]$,

$$
\begin{aligned}
& \mathcal{C}_{3}=\frac{e^{-\left(T_{c}+T_{n}\right) \sqrt{g / z_{c}}} \cdot L_{f 2}}{e^{\left(T_{c}+T_{n}\right) \sqrt{g / z_{c}}} \cdot e^{-T_{c} \sqrt{g / z_{c}}}-e^{-\left(T_{c}+T_{n}\right) \sqrt{g / z_{c}}} \cdot e^{T_{c} \sqrt{g / z_{c}}},} \\
& c_{4}=\frac{-e^{\left(T_{c}+T_{n}\right) \sqrt{g / z_{c}}} \cdot L_{f 2}}{e^{\left(T_{c}+T_{n}\right) \sqrt{g / z_{c}}} \cdot e^{-T_{c} \sqrt{g / z_{c}}}-e^{-\left(T_{c}+T_{n}\right) \sqrt{g / z_{c}}} \cdot e^{T_{c} \sqrt{g / z_{c}}}} \\
& t \in\left[T_{c}+T_{n}, 2 T_{c}\right], \\
& c_{3}=\frac{e^{-\left(T_{c}+T_{n}\right) \sqrt{g / z_{c}}} \cdot L_{f 2}}{e^{\left(T_{c}+T_{n}\right) \sqrt{g / z_{c}}} \cdot e^{-2 T_{c} \sqrt{g / z_{c}}}-e^{-\left(T_{c}+T_{n}\right) \sqrt{g / z_{c}}} \cdot e^{2 T_{c} \sqrt{g / z_{c}}}}, \\
& c_{4}=\frac{-e^{\left(T_{c}+T_{n}\right) \sqrt{g / z_{c}}} \cdot L_{f 2}}{e^{\left(T_{c}+T_{n}\right) \sqrt{g / z_{c}}} \cdot e^{-2 T_{c} \sqrt{g / z_{c}}}-e^{-\left(T_{c}+T_{n}\right) \sqrt{g / z_{c}}} \cdot e^{2 T_{c} \sqrt{g / z_{c}}} .} .
\end{aligned}
$$

$m$ is the mass of the whole biped lower limb; $g$ is the gravitational acceleration; $y$ is the displacement of the robot COM relative to the ground, which is opposite to the displacement of the ankle joint relative to the body fixed coordinate system in $Y_{c}$ direction; $\ddot{y}$ is the acceleration of the 
robot COM relative to the trunk fixed coordinate system in $Y_{\mathrm{c}}$ direction; $z_{c}$ is the absolute distance between the robot COM and the ground; $L_{f 1}$ is the absolute distance between $O_{1}$ and $O_{c}$ in $Y_{c}$ direction; $L_{f 2}$ is the absolute distance between $\mathrm{O}_{2}$ and $O_{c}$ in $Y_{c}$ direction. If the robot is symmetrical, then $L_{f 1}=$ $L_{f 2}=L_{f}$, actually if there is no sliding between the feet and the ground. $L_{f}$ is the maximum displacement of the trunk COM in the lateral plane, and we named it as maximum offset in the lateral plane.

3.3. Walking Pattern Generation in $X_{c}$ Direction. The direction changes of the trunk's acceleration will cause the loading sway back and forth. The larger the acceleration, the bigger the sway range is. In order to decrease this impact, the trunk is set to a constant velocity in $X_{c}$ direction (the COM adjustments change the velocity of the trunk slightly). That is to say, the ankle joint moves in an approximately constant velocity relative to the trunk fixed system, and then the trunk can keep a constant velocity relative to the ground. When the swing foot changes to the support foot, the swing foot should keep the same velocity as the trunk. With this constraint one of the easiest gait generation methods for swing leg is cubic polynomial fitting. One periodic motion contains two step periods.

In the first step period, the trajectory of the ankle joint of the swing leg relative to the trunk fixed coordinate system in $X_{c}$ direction is shown as follows:

$$
x_{s}=-\frac{8 D_{s}}{T_{c}^{3}} t^{3}+\frac{12 D_{s}-D_{d}}{T_{c}^{2}} t^{2}-\frac{2 D_{s}-D_{d}}{T_{c}} t-D_{s} .
$$

The trajectory of the ankle joint of the support leg relative to the trunk fixed coordinate system in $X_{c}$ direction is shown as follows:

$$
x=\frac{-2 D_{s}}{T_{c}} t+D_{s}
$$

where $x_{s}$ is the ankle displacement of the swing leg relative to the trunk fixed coordinate system in $X_{c}$ direction; $x$ is the ankle displacement of the support leg relative to the trunk fixed coordinate system in $X_{c}$ direction; $D_{s}$ is the step length of the ankle joint relative to the trunk fixed coordinate system in $X_{c}$ direction; $D_{d}$ is the increment of the step length between the two contiguous periods: if the step length increases, $D_{d}$ is a positive, and if the step length decreases, $D_{d}$ is a negative; $T_{c}$ is the step period.

The walking generation method in the second step period is the same as in the first step period, but the swing leg changes to the support leg and the support leg changes to the swing leg.

3.4. Walking Pattern Generation in $Z_{c}$ Direction. In order to decrease the impact, when the swing foot hit the ground and changes to the support foot, the following two conditions are needed:

(1) the ideal dropping velocity in $Z_{c}$ direction is zero;

(2) the raise velocity decreases to zero when the swing foot reaches the planned raise height.

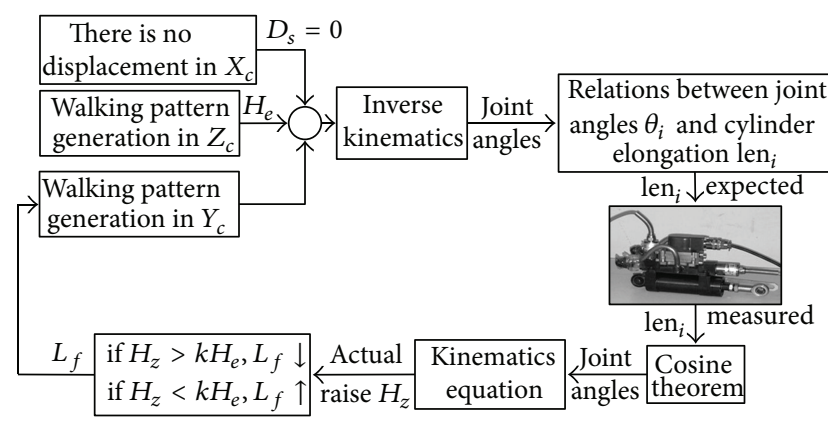

Figure 5: Diagram of the motion adjustment in $Y_{c}$ direction.

With the constraints, cubic polynomial fitting can be used here. In the first step period, the trajectory of the ankle in the swing leg relative to the trunk in $Z_{c}$ direction is shown as follows:

$$
\begin{gathered}
t \in\left[0, \frac{T_{c}}{2}\right], \quad H_{z}=-\frac{16 H_{n}}{T_{c}^{3}} t^{3}+\frac{12 H_{n}}{T_{c}^{2}} t^{2}, \\
t \in\left[\frac{T_{c}}{2}, T_{c}\right], \quad H_{z}=\frac{16 H_{n}}{T_{c}^{3}} t^{3}-\frac{36 H_{n}}{T_{c}^{2}} t^{2}+\frac{24 H_{n}}{T_{c}} t-4 H_{n},
\end{gathered}
$$

where, $H_{z}$ is the raise height of the swing leg; $H_{n}$ is the expected maximum raise height of the ankle joint in the swing leg relative to the trunk fixed coordinate system in $Z_{c}$ direction; $T_{c}$ is the step period.

The support foot keeps on touch with the terrain. In the second step period, the swing leg changes to the support foot.

\section{Balance Control}

4.1. Motion Adjustment in $Y_{c}$ Direction. From (3), (6), We can see that $c_{1}, c_{2}, c_{3}$, and $c_{4}$ are constants, which are relative to $z_{c}, T_{c}$, and $L_{f}$.

As mentioned above, $T_{c}$ is the step period; $z_{c}$ is the absolute distance between the robot COM and the ground, and we can obtain $z_{c}$ from experiments, but here we use the absolute distance between the origin point of the trunk fixed coordinate system and the ground $z$ to replace, which is easier to obtain. The difference is compensated by adjusting $L_{f}$, with a different $L_{f}$, the trunk's acceleration, velocity, and displacement in $Y_{c}$ direction changed accordingly. From the marking time (walking but the step length is zero) experiments, we may obtain the relationship between the loading weight, step period, and $L_{f}$, which could be retained in the following movements.

When adjusting $L_{f}$ (shown as Figure 5), we care for the actual raise height $H_{z}$ of the swing leg rather than ZMP position in $Y_{c}$ direction (that is to say, we do not care whether the equilibrium point $\mathrm{O}_{1}$ or $\mathrm{O}_{2}$ is in or out the convex polygon composed by the support foot). For the robot without active rolling joint in the ankle, the roll motion of the trunk is inevitable. The actual raise height $H_{z}$ of the swing leg is larger than the planned $H_{e}$. We should guarantee that $H_{z} \approx k \cdot H_{e}(k$ is more than one and obtained from experiments. It is related 


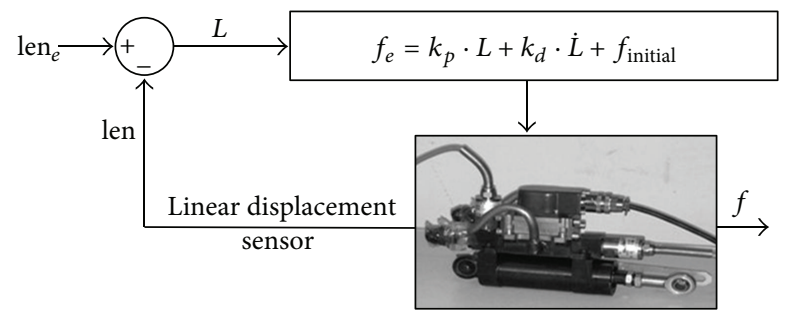

FIgure 6: Position servo.

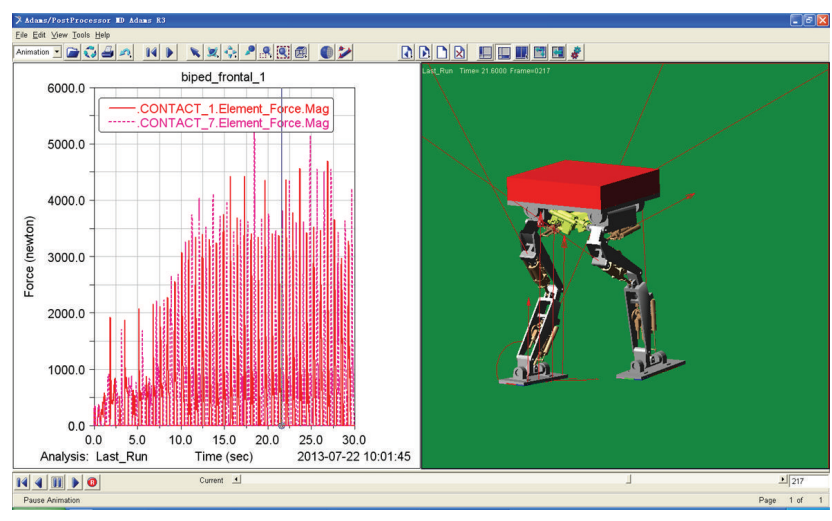

Figure 7: Diagram of the ground reaction force.

to the robot system and has little with the loading weight or the motion characteristics of the robot).

(i) If $L_{f}$ is too small the swing leg cannot leave the ground normally or the robot topples toward the swing leg then the actual raise height $H_{z}$ of the swing leg is less than the expected raise height $k \cdot H_{e}$;

(ii) If $L_{f}$ is too large, the robot topples toward the support leg $H_{z}$ and the actual raise height is larger than $k \cdot H_{e}$.

4.2. Motion Control in the Frontal Plane. With the motion adjustment in the lateral plane, the robot walking system can walk front and back, left and right with a small step length. In order to increase the impact resistance, cylinders in the frontal plane are controlled by the hydraulic actuated force $f_{e}$ which is used to servo the elongation $\operatorname{len}_{e}$ of the hydraulic cylinder. Shown in Figure $6, f_{\text {initial }}$ is used to meet the offset, and $k_{p}$ and $k_{d}$ are obtained from experiments.

4.3. Balance Control on Uneven Terrain. Unlike human size biped robot, when the hydraulic actuated biped robot with load walks dynamically, the ground reaction force distributed unevenly and varied in a large dynamic range (shown as Figure 7). The ground reaction force to the physical prototype is smaller in a certain extent, because there is elastic mechanism on the feet which provides a buffer.

ZMP information for the biped robot walking on the uneven terrain is important, but it is hard to calculate it precisely. Obviously, we can obtain it by six-dimensional force sensor. But the larger the necessary measure range is, the more expensive the sensor is.

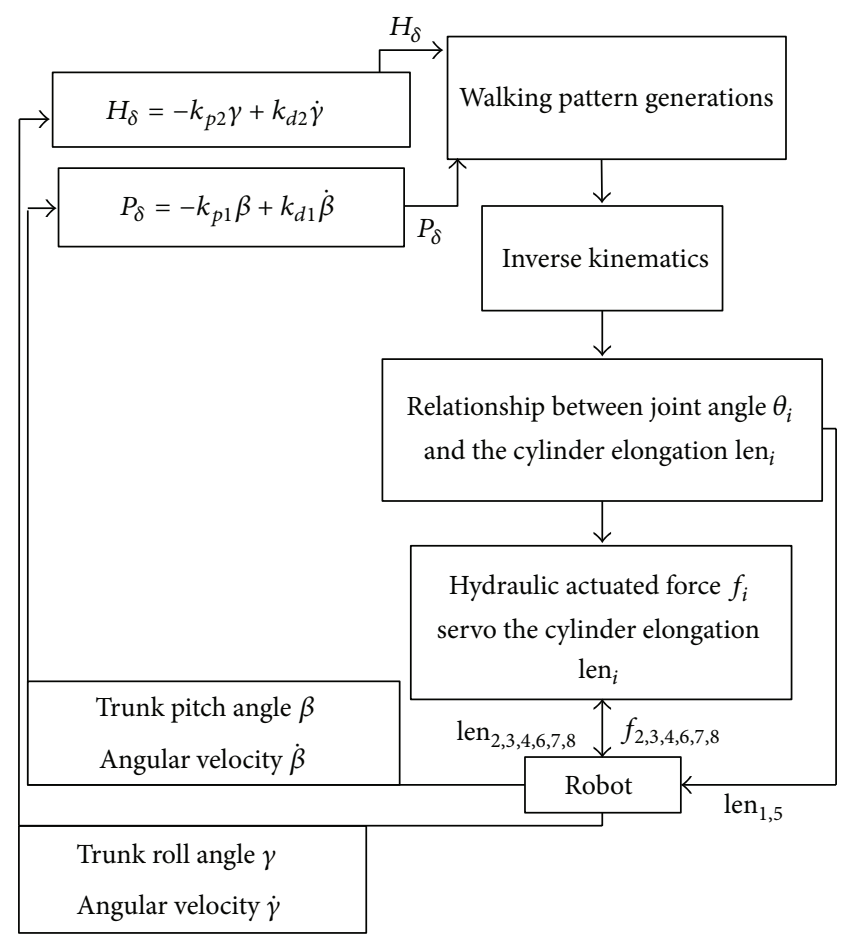

FIGURE 8: Structure of balance controller on uneven terrain.

From the kinetic characteristics of the biped robot lower limb we find that trunk attitude, especially the pitch angle and the roll angle, could be used as controlled variable. In the actual environment, the roll and pitch movements of the trunk cannot be eliminated at least for the robot with foot, but without active roll joint in the ankle. We use this information to realize the stable walking of the biped robot lower limb (shown in Figure 8).

(1) When the trunk rolls along $X_{c}$ in an active angle, the length of the right leg is shorter than expected, while the left one is longer than expected, we should increase the length of the right leg and decrease the length of the left leg. But the adjustment amount $H_{\delta}$ is the same, which is relative to the roll angle $\gamma$ and the angular velocity $\dot{\gamma}$ of the trunk; when the trunk rolls along $X_{c}$ in a negative angle, and we should decrease the length of the right leg and increase the length of the left leg accordingly.

(2) When the trunk rolls along $Y_{c}$ in an active angle, the trunk tends to fall forward; in particular the robot walks down slope. We should move the COM backward relative to the ground (the absolute displacement of the support leg $x$ relative to the trunk fixed coordinate system increases $P_{\delta}$ ); when the trunk turns along $Y_{c}$ in a negative angle, the trunk tends to fall backward, and in particular the robot walks upslope. Then we should move the COM forward (the absolute displacement of the support leg $x$ relative to the trunk fixed coordinate system decreases $\left.P_{\delta}\right) . P_{\delta}$ is relative to the pitch angle $\beta$ and angular velocity $\dot{\beta}$ 


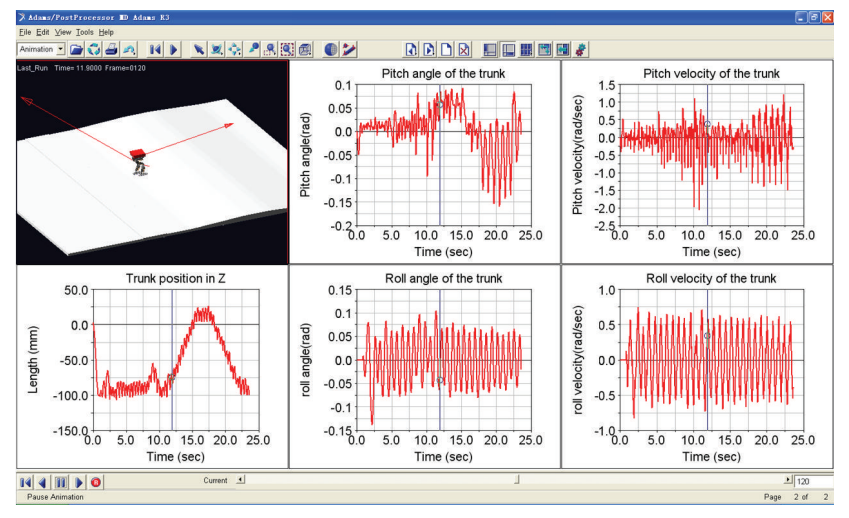

FIGURE 9: Walking with the balance control method on uneven terrain.

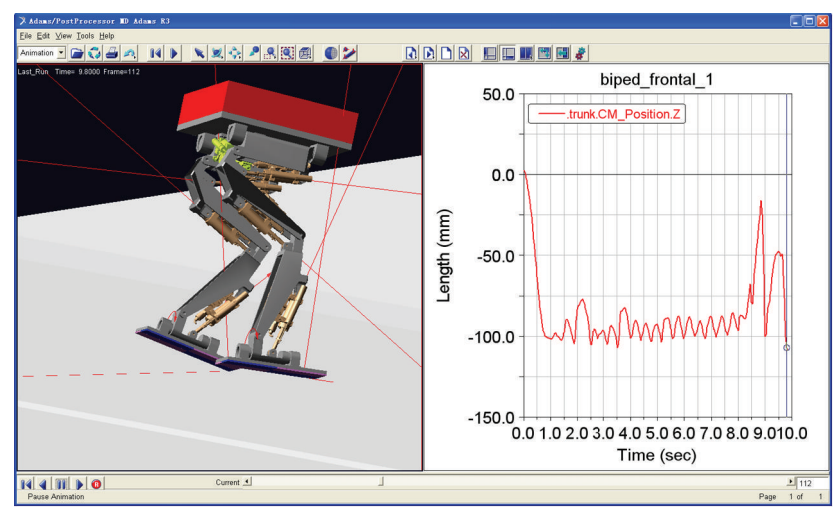

FIGURE 10: Walking without the balance control method on uneven terrain.

of the trunk (shown in Figure 8). It is important that $k_{p 1}, k_{d 1}, k_{p 2}$, and $k_{d 2}$, are determined by the biped robot lower limb itself, they have little to do with the loading weight and the step period. $k_{p 1}, k_{d 1}, k_{p 2}$, and $k_{d 2}$ are obtained from simulation experiments and have some changes in the physical prototype experiments.

\section{Experimental Verification}

5.1. Simulation Experiments. It is assumed that the terrain information has been contained in the walking generation. If the step height is less than $20 \mathrm{~mm}$ or the gradient of the slope is less than $\pm 3^{\circ}$, the road could be seen as flat road. But we should know when the robot walks up/down steps, because the robot does not have toe, and then it is forbidden that less than half of the support foot is on the step.

The robot loads a block whose weight is $100 \mathrm{kgf}$. Walking across a step which is $20 \mathrm{~mm}$ in height and two slopes (the gradients are $3^{\circ}$ and $-3^{\circ}$, resp.). At the same time there are two disturbance forces on the center of the loading pointing to $X_{c}$ and $Y_{c}$, respectively. The disturbances are added manually by two cosine waves whose amplitude is $40 \mathrm{~N}$. With the proposed adjusting method, the biped robot lower limb walks on the uneven terrain under disturbance successfully (shown in Figure 9). When walking without the balance control

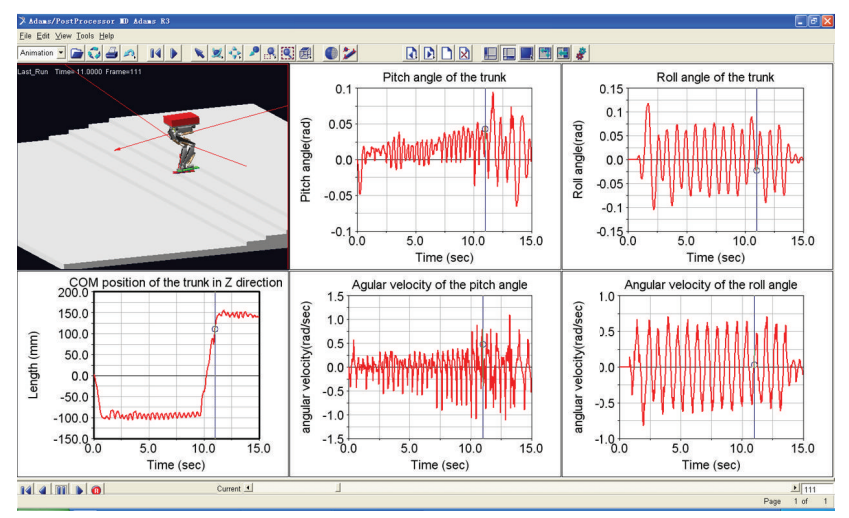

FIGURE 11: Walking up steps.

method proposed in this paper the robot cannot even pass the step which is $20 \mathrm{~mm}$ in height (shown in Figure 10). For the robot having big foot which is $270 \mathrm{~mm}$ in length, when the robot walks on steps, it is advised to use bigger step length, but when the robot walk on slopes, it is advised to use smaller step length. For example, when the robot walking on slopes the step length is $240 \mathrm{~mm}$ and the step period is $0.4 \mathrm{~s}$ (shown in Figures 9 and 10); when walking up steps which is $60 \mathrm{~mm}$, the step length is $300 \mathrm{~mm}$ and the step period is $0.4 \mathrm{~s}$ (shown in Figure 11).

5.2. Physical Prototype Experiments. Without power producer, the weight of the original robot is $40 \mathrm{~kg}$, but the mass of one single leg is about $14 \mathrm{~kg}$, and the leg's mass cannot be ignored. Obviously, it is not fit for the assumed condition of LIPM. With the "dynamic equilibrium method," the biped lower-limb can walk along, walk back, and walk in the lateral plane successfully. With the proposed control method in the paper, the robot pass through a carpet which is about $10 \mathrm{~mm}$ in thickness, a rubber blanket which is about $8 \mathrm{~mm}$ in thickness, and a plank which is about $13 \mathrm{~mm}$ in thickness successfully (shown in Figure 12). For the robot walking without load, the robot mass is concentrated on the legs. Then the position changes of the leg may cause body COM position changing obviously, so the step length of the physical prototype in frontal plane is $60 \mathrm{~mm}$ and the step period is $0.45 \mathrm{~s}$.

\section{Conclusion}

Human size biped robot actuated by electrical motor developed rapidly and became mature gradually. BigDog [17] and Petman [18] made by Boston Dynamics bring hydraulic driving technology to robot study again. The paper gives an outline of a hydraulic actuated biped robot lower limb with load ability. The biped robot lower limb only has 8 active freedoms, for each leg there are four, two in the hip, one in the knee and one in the ankle.

3D-LIPM with ZMP criterion is widely used in the biped robot control. Considering the boundedness of the LIPM model and the complexity of ZMP, the paper proposes an easy walking generation method and balance control method. 

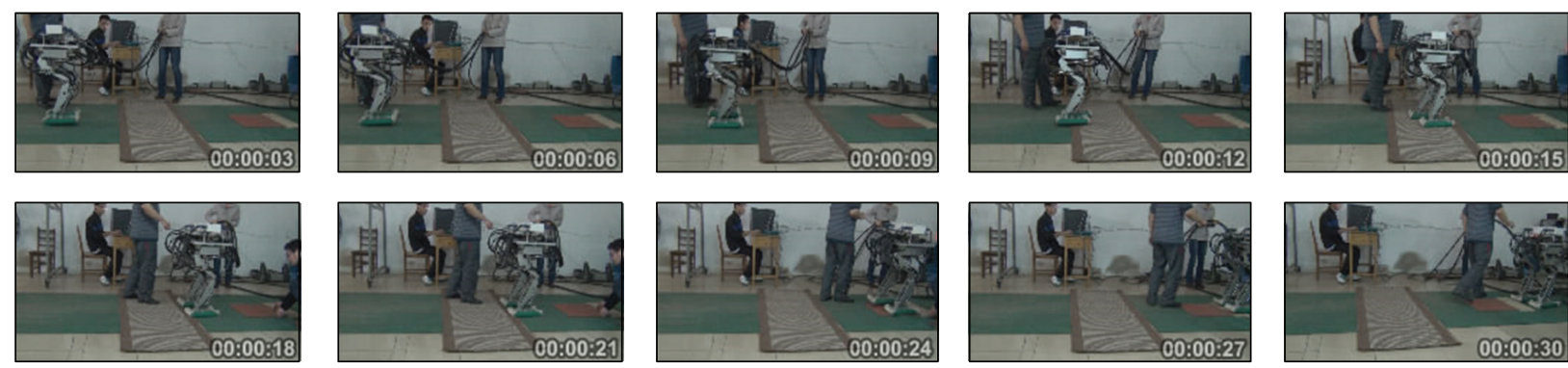

FIGURE 12: Physical prototype experiments.

"Dynamic equilibrium method" and the cubic polynomial fitting method are used in the walking generation. This method is fit for both the active and underactive biped robots with foot. Not as LIMP, "dynamic equilibrium method" proposed in this paper has no limit to the mass distribution between the trunk and the legs. The paper also proposes a simple control method without using the ZMP information directly. The biped robot lower-limb adjusts the maximum offset in the lateral plane according to the actual raise height of the swing leg in the marking time; the biped robot adjusts the COM position in $X_{c}$ direction, according to the pitch angle and angular velocity without using ZMP information directly.

With the proposed method the biped robot lower limb with a box weight $100 \mathrm{kgf}$ can walk stably on uneven terrain under the disturbance whose amplitude is $40 \mathrm{~N}$. The control method proposed in this paper is also effective to the hydraulic actuated biped robot lower limb which does not fit for the assumed conditions of LIPM. When weighting empty, with the proposed method in the paper, the hydraulic actuated biped robot lower limb can walk on the uneven terrain successfully.

\section{Acknowledgments}

This work is supported by the Independent Innovation Foundation of Shandong University with Grant (2011JC011) and the State Key Program of National Natural Science of China (61233014).

\section{References}

[1] M. Vukobratović and B. Borovac, "Zero-moment point-thirty five years of its life," International Journal of Humanoid Robotics, vol. 1, pp. 157-173, 2004.

[2] S. Kajita, F. Kanehiro, K. Kaneko et al., "Biped walking pattern generation by using preview control of zero-moment point," in Proceedings of the IEEE International Conference on Robotics and Automation (ICRA '03), vol. 2, pp. 1620-1626, September 2003.

[3] K. Kaneko, F. Kanehiro, S. Kajita et al., "Humanoid robot HRP-2," in Proceedings of the IEEE International Conference on Robotics and Automation (ICRA '04), pp. 1083-1090, May 2004.

[4] J. H. Kim, "Walking pattern generation of a biped walking robot using convolution sum," in Proceedings of the 7th IEEE-RAS International Conference on Humanoid Robots, pp. 539-544, December 2007.
[5] J. H. Kim, J. Y. Kim, and J. H. Oh, "Adaptive walking pattern generation and balance control of the passenger-carrying biped robot, HUBO FX-1, for variable passenger weights," Autonomous Robots, vol. 30, no. 4, pp. 427-443, 2011.

[6] M. Hirose and K. Ogawa, "Honda humanoid robots development," Philosophical Transactions of the Royal Society A, vol. 365, no. 1850, pp. 11-19, 2007.

[7] W. Suleiman, F. Kanehiro, K. Miura, and E. Yoshida, "Generating dynamically stable walking patterns for humanoid robots using quadratic system model," in Proceedings of the IEEE/ ASME International Conference on Advanced Intelligent Mechatronics (AIM '10), pp. 645-651, July 2010.

[8] J.-H. Kim, J.-Y. Kim, and J.-H. Oh, "Adjustment of home posture of a biped humanoid robot using an inertial sensor and force torque sensors," in Proceedings of the IEEE/RSJ International Conference on Intelligent Robots and Systems (IROS '07), pp. 2223-2229, November 2007.

[9] J. Yang, Q. Huang, J. Li, C. Li, and K. Li, "Walking pattern generation for humanoid robot considering upper body motion," in Proceedings of the IEEE/RSJ International Conference on Intelligent Robots and Systems (IROS '06), pp. 4441-4446, October 2006.

[10] Q. Huang, K. Yokoi, S. Kajita et al., "Planning walking patterns for a biped robot," IEEE Transactions on Robotics and Automation, vol. 17, no. 3, pp. 280-289, 2001.

[11] Y. Sugahara, T. Hosobata, Y. Mikuriya, H. Sunazuka, H.-O. Lim, and A. Takanishi, "Realization of dynamic human-carrying walking by a biped locomotor," in Proceedings of the IEEE International Conference on Robotics and Automation (ICRA '04), pp. 3055-3060, May 2004.

[12] K. Hashimoto, A. Hayashi, T. Sawato et al., "Terrain-adaptive control to reduce landing impact force for human-carrying biped robot," in Proceedings of the IEEE/ASME International Conference on Advanced Intelligent Mechatronics (AIM '09), pp. 174-179, July 2009.

[13] Toyota Motor Corporation, http://www.toyota-global.com/innovation/partner_robot/aichi_expo_2005/index03.html.

[14] X. Rong, Y. Li, J. Ruan, and B. Li, "Design and simulation for a hydraulic actuated quadruped robot," Journal of Mechanical Science and Technology, vol. 26, no. 4, pp. 1171-1177, 2012.

[15] Z. Zhang, Q. Huang, G. R. Li, Y. C. Zhao, and W. M. Zhang, "Kinematics analysis and motion planning of a humanoid leg with 7DOF and double spherical hip joint," Robot, vol. 6, p. 7, 2007.

[16] J.-Y. Kim, I.-W. Park, and J.-H. Oh, "Walking control algorithm of biped humanoid robot on uneven and inclined floor," Journal of Intelligent and Robotic Systems, vol. 48, no. 4, pp. 457-484, 2007. 
[17] Boston Dynamics, http://www.bostondynamics.com/robot_ bigdog.html.

[18] Boston Dynamics, http://www.bostondynamics.com/robot_ petman.html. 

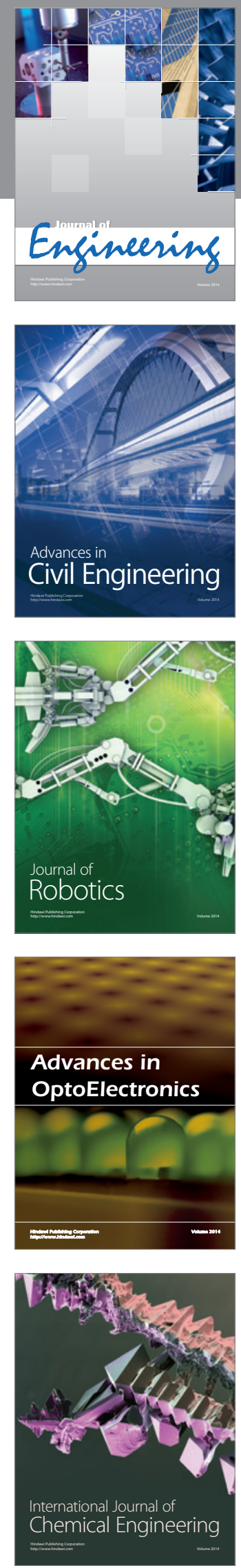

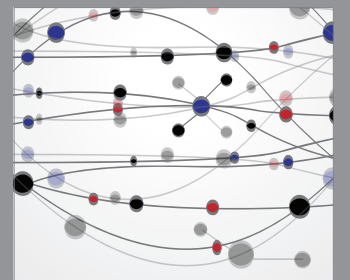

The Scientific World Journal
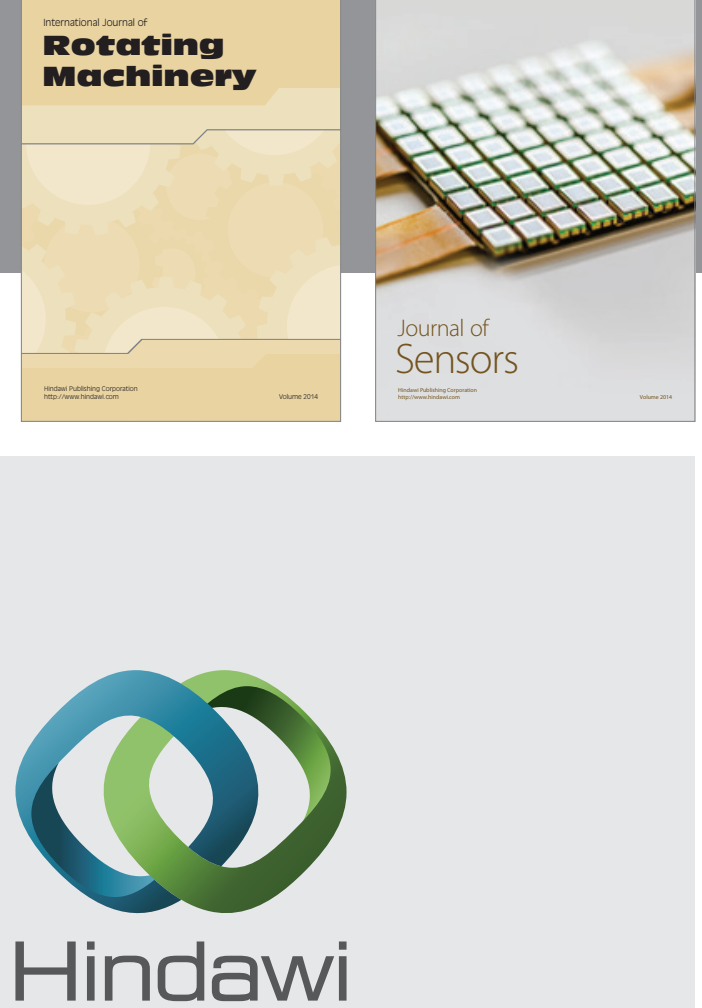

Submit your manuscripts at http://www.hindawi.com
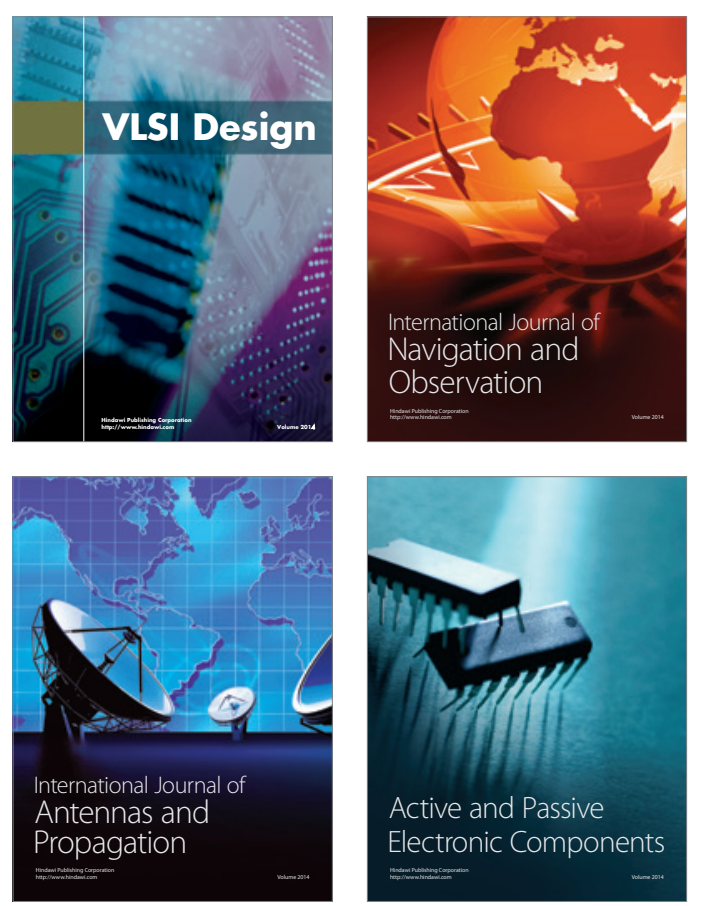
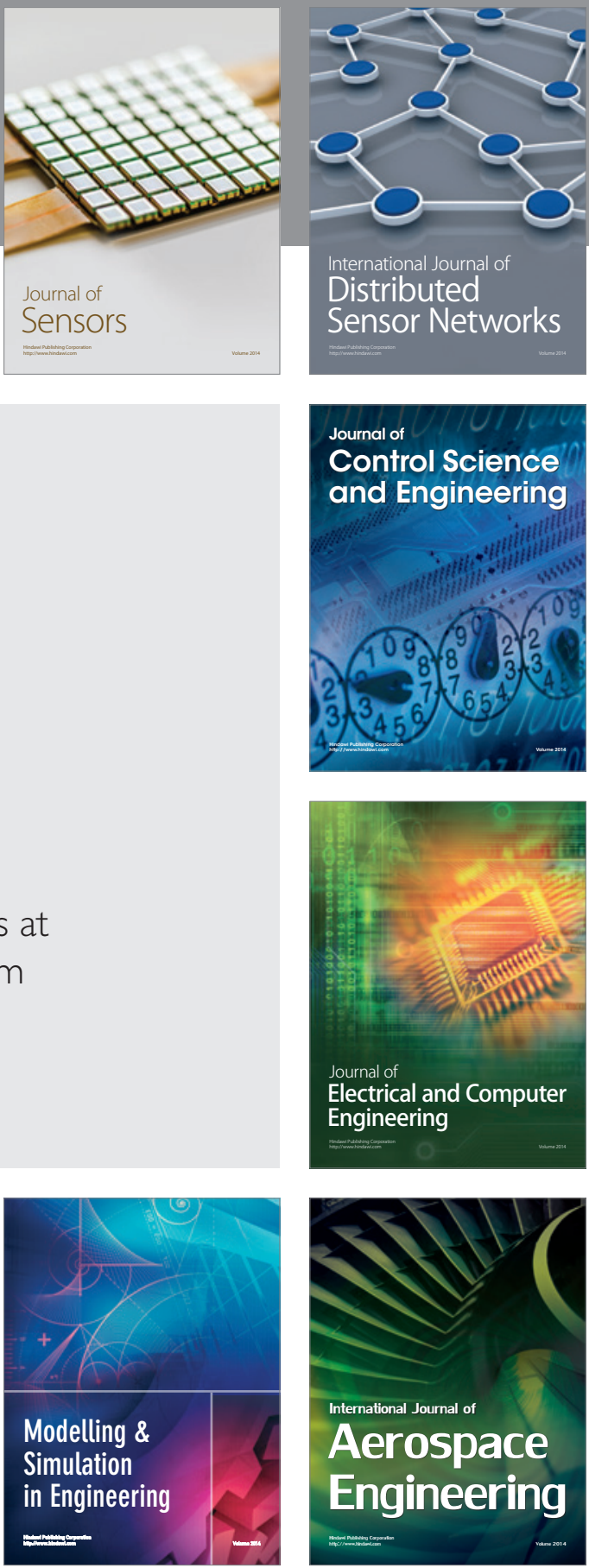

Journal of

Control Science

and Engineering
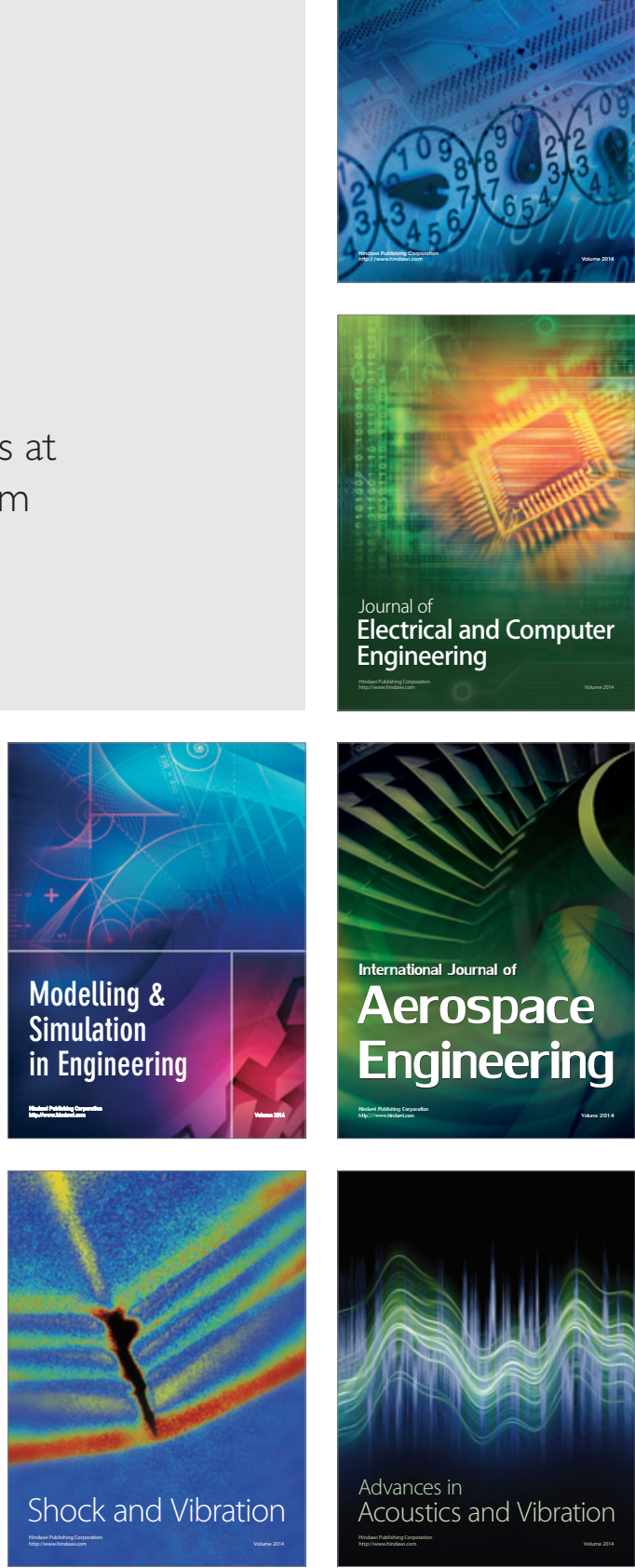